\title{
ANALYSIS OF CROSS-FLOW INDUCED VIBRATION IN AN INLINE AND STAGGERED CONFIGURATION
}

\author{
Vimal D. Tandel ${ }^{1}$, Rajesh V. Patil ${ }^{2}$ \\ ${ }^{I} P G$ Scholar, Department of Mechanical Engineering SITS, Pune, Maharashtra, India \\ ${ }^{2}$ Asst. Professor, Department of Mechanical Engineering SITS, Pune, Maharashtra, India
}

\begin{abstract}
In many engineering applications like heat exchanger, radiator, evaporator, nuclear power plant and thermal power plant, arrangement of tubes is very crucial. Fluid elastic instability forms the basis for deciding the type of arrangement and tube spacing but the phenomenon of vortex induced vibration is random in nature. Tube spacing also plays a critical role in different types of arrangement. Different type of application requires different tube spacing and the range of tube spacing vary from 1 to 6. Vortex Induced Vibration in cross flow around the inline and staggered arrangement of the tube arrays is experimentally studied for varying P/d (tube spacing) ratio. It is observed that with the increase in the velocity, the amplitude displacement increases. As the amplitude displacement of the tube reduces, the pitch over diameter ratio is increased from 2 to 4 . It is also observed that between inline and staggered arrangement, the amplitude displacement of staggered arrangement is more compared to inline arrangement for same tube spacing.
\end{abstract}

Keywords: Vortex Induced Vibration, Inline Arrangement, Staggered Arrangement, Regression Analysis

\section{INTRODUCTION}

The interaction between the fluid flow and the cylindrical structures play an important role in many applications of engineering. Due to its practical importance, flow around the cylindrical structures has been studied. The fluid flow around the cylindrical structures exhibit various physical phenomena like turbulence buffeting, vortex induced vibration, fluid-elastic instability and acoustic resonance which may severely damage the cylindrical structures. In heat exchanger, the cylindrical tubes are placed in close proximity to one another and there are large numbers of tubes. The forces experienced by the cylindrical tubes and the flow field around the tubes are entirely different compared to the single tube immersed in the fluid flow. Vortex induced vibration is the major consideration since heat exchanger are designed keeping in mind the threshold for fluid-elastic instability.

Grover and Weaver [10] used a 19-tube array with only one tube flexible and the other was made rigid. They found out that vibrations induced by the fluid flow were responsible for the instability of the single flexible cylinder. They concluded that the mechanism which was responsible for the instability of a single flexible cylinder is the same mechanism leading to the instability of an entire flexible array. Chunlei et al [6] studied numerically the vortex shedding characteristic and forces acting on the tube in an inline cylinder array. They considered flow to be laminar and studied the effect of tube spacing on the array. Computations were carried out for a six row inline tube bank. They concluded that by increasing the spacing makes the flow more asymmetric and induces vortex shedding. Vortex shedding starts from the last cylinder and proceeding upstream. E. Longatte et al. [8] studied the numerical methods for investigating the effects of flow induced vibration on the tube bundle motion in presence of fluid at rest and single phase cross-flows. They used arbitrary Langrange Euler formulation for the fluid computation to simulate thermo-hydraulics and mechanics. The main purpose was to provide a numerical estimate of the critical flow velocity for the threshold of fluid-elastic instability of tube bundle without experimental investigation. They validated their results with the available experimental data obtained with same configuration. S. Pasto [13] performed the experiment on freely vibrating circular cylinder in turbulent and laminar flows. He performed the experiment in the wind tunnel by varying the roughness of the cylinder and mass damping parameter and studied the behavior of the cylinder in terms of Reynolds number.

\section{EXPERIMENTAL DETAILS}

Vortex induced vibration is a very complex phenomena and the vibration amplitude of the tube is affected by many parameters. In order to find out the amplitude displacement of the flexible tube for vortex induced vibration phenomena, many experiments have been performed. In this paper, effect of tube spacing, i.e. pitch over diameter ratio, is found out experimentally. Experiments were conducted on wind tunnel where maximum attainable velocity was $25 \mathrm{~m} / \mathrm{s}$. Effect of pitch over diameter ratio has been found out for both the inline and staggered arrangement. Since many applications of engineering lie below the ratio, the pitch over diameter has been selected 2, 3 and 4.The tube end is supported at the top surface of the test section of wind tunnel and the other end is free. Cantilever tube bundles are subjected to air flow. The support has been taken cantilever because natural frequency of cantilever tube is very less as compared to the other type of end supports and hence its stiffness. Thus, it maintains the less difference between the natural frequency and the forcing frequency of the tubes. 


\subsection{Sample Tested}

The sample tested in the tunnel is an array of circular hollow cylinder $275 \mathrm{~mm}$ long with a diameter of $10 \mathrm{~mm}$ and its inner diameter is $9.5 \mathrm{~mm}$. The material selected for the tube is Copper. The sample has been transversely placed with regard to the wind direction from the top section of the tunnel. The sample contains of an array of 9 cylinders placed in a $3 \times 3$ configuration for inline arrangement and an array of 8 cylinders arranged in staggered configuration to form a staggered arrangement. Of all the nine cylinders, only the cylinder in the center of the first row is flexible while the other cylinders are made rigid for inline arrangement. For staggered arrangement, one of the tubes in the middle row is flexible.

\subsection{Arrangement of Tubes}

For carrying out this experiment, two different types of arrangement are mainly used, namely staggered and inline arrangement. In present case, experiments are carried out on an inline arrangement. The sample contains of an array of 9 cylinders placed in a $3 \times 3$ configuration. Of all the nine cylinders, only the cylinder in the center of the front row is flexible while the other cylinders are made rigid. For inline arrangement, readings are taken at the center tube which is flexible. For staggered arrangement, one of the tubes in the middle row is kept flexible; amplitude displacement is measured at this flexible tube.

\subsection{Experimental Setup}

The wind tunnel is used to carry the experiment. The block diagram of the wind tunnel is represented by figure 3 . The wind tunnel has a cross section $30 \mathrm{~cm}$ wide and $30 \mathrm{~cm}$ high. The global length of the tunnel, from the inlet to the end of the tunnel, is about $1000 \mathrm{~cm}$. The values of the velocities are obtained with the help of Pitot tube and anemometer arrangement. The maximum velocity attainable is $25 \mathrm{~m} / \mathrm{s}$. For the experiments carried out, velocity was varied from 0 $-20 \mathrm{~m} / \mathrm{s}$.

Two different types of arrangement of tube arrays, i.e. inline and staggered are used to carry out the experiments. For inline arrangement, nine tubes are used and the amplitude displacement of the center tube is found out with the help of the load cell arrangement. Eight tubes are used for staggered arrangement and the amplitude displacement of the tube on the right side from the direction of the flow is obtained. The displacement amplitude of the tube is indicated by the strain indicator which is connected with the load cell. Results obtained from the experiments performed for both the arrangements are shown in the table 1 and 2. The data that has been collected from the experiments are represented in the form of graphs and the results are concluded from the graphs.

Table -1: Experimental Results for Inline Arrangement

\begin{tabular}{|c|c|c|c|c|c|c|}
\hline \multirow{3}{*}{$\begin{array}{l}\text { Parameters / } \\
\text { S.No. }\end{array}$} & \multicolumn{6}{|c|}{ Pitch over diameter ratio } \\
\hline & \multicolumn{2}{|l|}{2} & \multicolumn{2}{|l|}{3} & \multicolumn{2}{|l|}{4} \\
\hline & $\begin{array}{l}\text { Velocity } \\
(\mathrm{m} / \mathrm{s})\end{array}$ & $\begin{array}{l}\text { Displacement } \\
(\mu \varepsilon)\end{array}$ & $\begin{array}{l}\text { Velocity } \\
(\mathrm{m} / \mathrm{s})\end{array}$ & $\begin{array}{l}\text { Displacement } \\
(\mu \varepsilon)\end{array}$ & $\begin{array}{l}\text { Velocity } \\
(\mathrm{m} / \mathrm{s})\end{array}$ & $\begin{array}{l}\text { Displacement } \\
(\mu \varepsilon)\end{array}$ \\
\hline 1 & 5 & 4 & 5 & 3 & 5 & 2 \\
\hline 2 & 10 & 7 & 10 & 5 & 10 & 3 \\
\hline 3 & 15 & 9 & 15 & 8 & 15 & 6 \\
\hline 4 & 20 & 13 & 20 & 11 & 20 & 9 \\
\hline
\end{tabular}

Table -2: Experimental Results for Inline Arrangement

\begin{tabular}{|c|c|c|c|c|c|c|}
\hline \multirow[b]{3}{*}{$\begin{array}{l}\text { Parameters } \\
\text { / S.No. }\end{array}$} & \multicolumn{6}{|c|}{ Pitch over diameter ratio } \\
\hline & \multicolumn{2}{|l|}{2} & \multicolumn{2}{|l|}{3} & \multicolumn{2}{|l|}{4} \\
\hline & $\begin{array}{l}\text { Velocity } \\
(\mathrm{m} / \mathrm{s})\end{array}$ & $\begin{array}{l}\text { Displaceme } \\
\text { nt } \\
(\mu \varepsilon)\end{array}$ & $\begin{array}{l}\text { Velocity } \\
(\mathrm{m} / \mathrm{s})\end{array}$ & $\begin{array}{l}\text { Displaceme } \\
\mathrm{nt} \\
(\mu \varepsilon)\end{array}$ & $\begin{array}{l}\text { Velocity } \\
(\mathrm{m} / \mathrm{s})\end{array}$ & $\begin{array}{l}\text { Displaceme } \\
\mathrm{nt} \\
(\mu \varepsilon)\end{array}$ \\
\hline 1 & 5 & 5 & 5 & 4 & 5 & 3 \\
\hline 2 & 10 & 7 & 10 & 6 & 10 & 5 \\
\hline 3 & 15 & 11 & 15 & 9 & 15 & 9 \\
\hline 4 & 20 & 15 & 20 & 12 & 20 & 11 \\
\hline
\end{tabular}

For inline arrangement, graph of Amplitude displacement versus velocity for different $\mathrm{P} / \mathrm{d}$ ratio has been shown in figure 4. As observed from the graph, for $\mathrm{P} / \mathrm{d}$ ratio $=2$, displacement amplitude is maximum compared to $\mathrm{P} / \mathrm{d}$ ratio $=3$ and $\mathrm{P} / \mathrm{d}$ ratio $=4$. Also, it is observed that, with the increase in the velocity the amplitude displacement of the tube increases. 


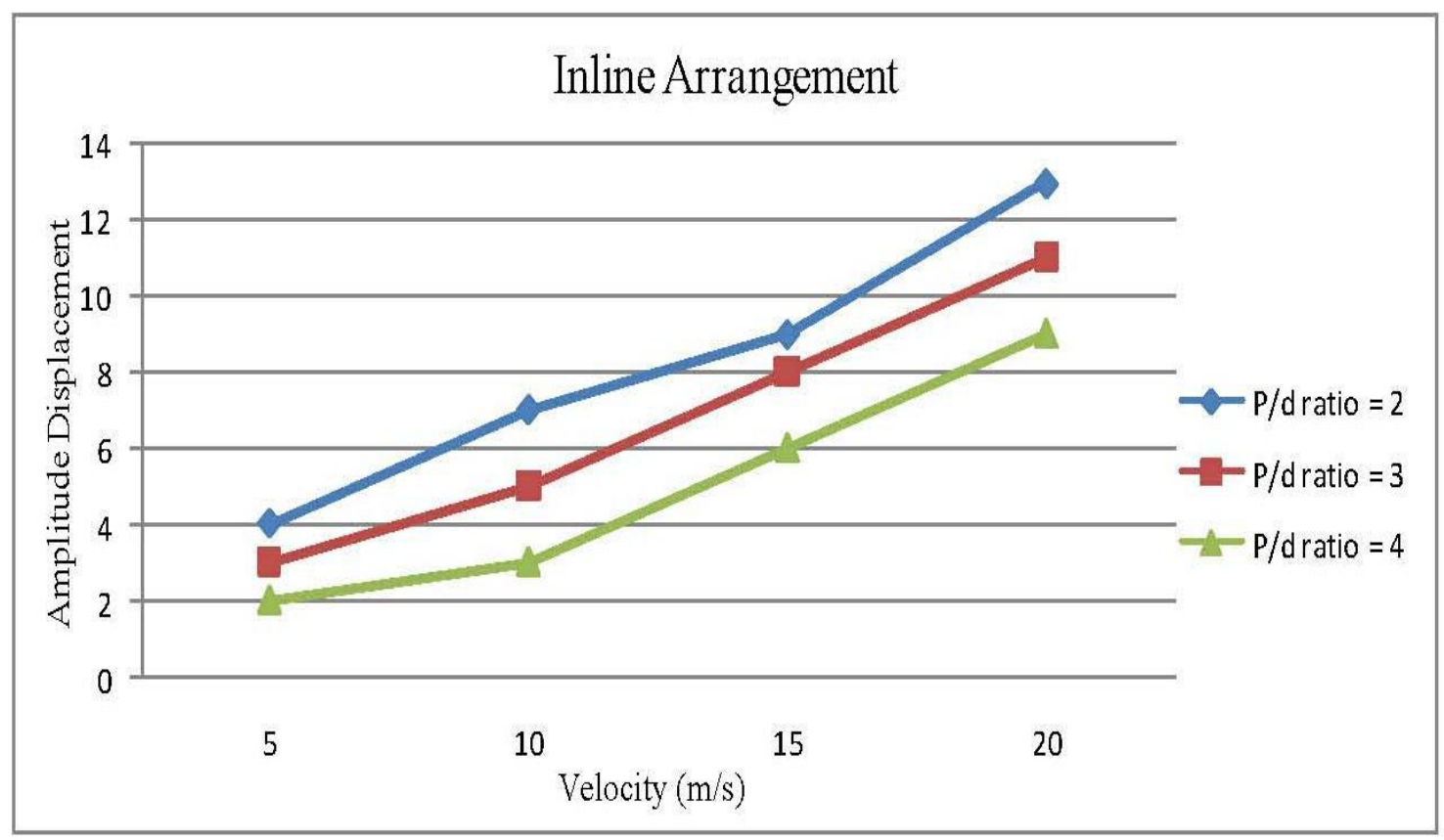

Chart -1: Displacement Amplitude of the flexible tube for inline arrangement

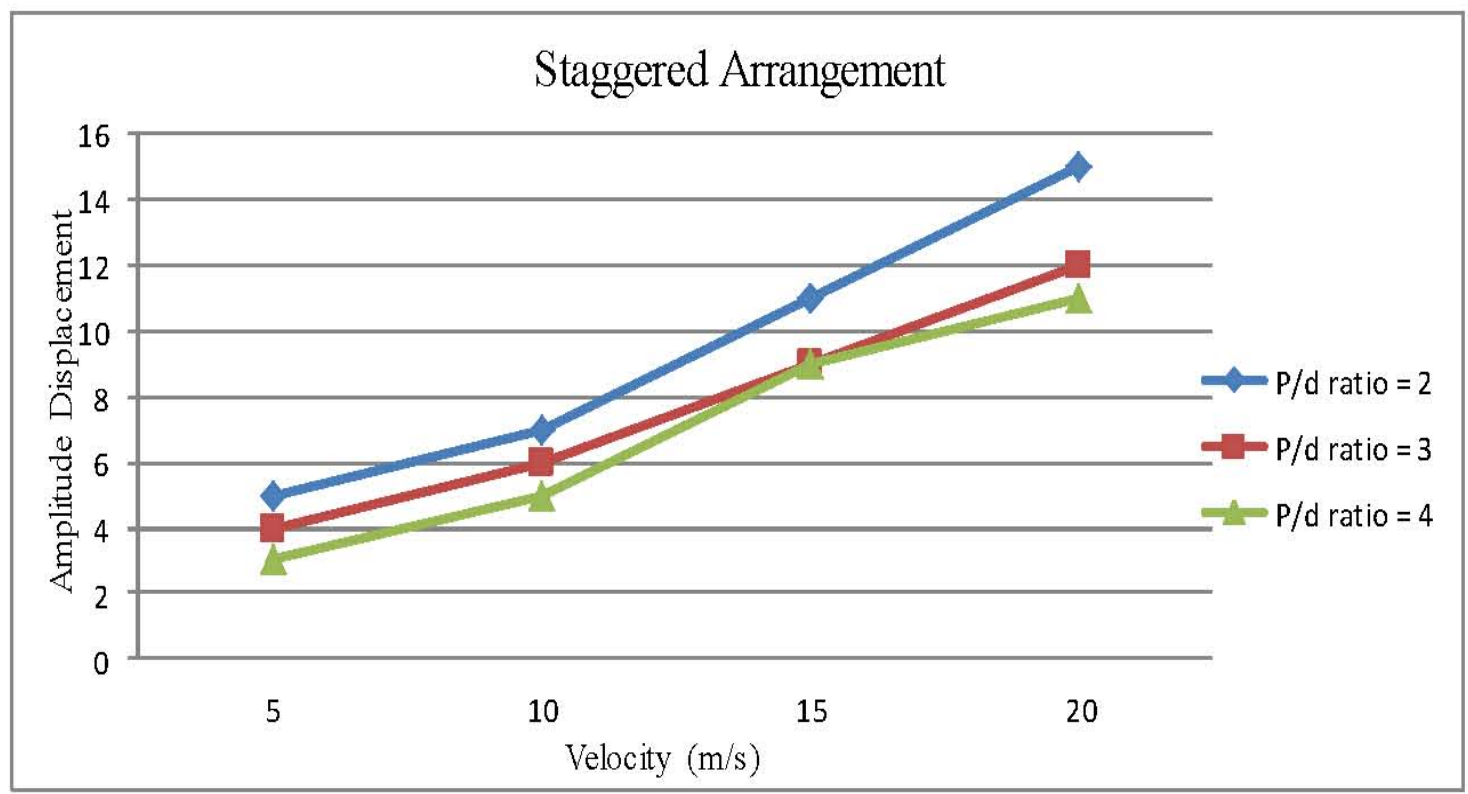

Chart -2: Displacement Amplitude of the flexible tube for staggered arrangement

For staggered arrangement, graph of Amplitude displacement versus velocity for different $\mathrm{P} / \mathrm{d}$ ratio has been shown in figure 5 . As observed from the graph, for $\mathrm{P} / \mathrm{d}$ ratio

$=2$, displacement amplitude is maximum compared to $\mathrm{P} / \mathrm{d}$ ratio $=3$ and $\mathrm{P} / \mathrm{d}$ ratio $=4$. Similar conclusions can be derived from this graph, but the amplitude displacement of staggered arrangement is more compared to inline arrangement.

Based on the results obtained from the experiments conducted, it can be observed that for For inline arrangement, maximum amplitude displacement is measured for the $\mathrm{p} / \mathrm{d}$ ratio equal to 2 . The value of amplitude displacement goes on decreasing as the value of $\mathrm{p} / \mathrm{d}$ ratio increases from 2 to 4.It can be seen that for staggered arrangement, results obtained are similar to inline arrangement i.e. the maximum value is measured for $\mathrm{p} / \mathrm{d}$ ratio equal to 2 and the value of displacement goes on decreasing as the $\mathrm{p} / \mathrm{d}$ ratio increases from 2 to 4 . But when the results are compared for staggered and inline arrangement, the value of displacement amplitude for staggered arrangement is more compared to that of inline arrangement. As seen in the table, for $\mathrm{P} / \mathrm{D}$ ratio $=2$, the maximum value of displacement amplitude is $15 \mu \varepsilon$ for staggered arrangement while for inline arrangement the maximum value is $13 \mu \varepsilon$. 


\section{REGRESSION ANALYSIS}

Regression analysis is a statistical tool for investigation of relationship between the variables. Regression analysis tool is used to understand the statistical dependence of one variable on other variable. When the effect of multiple variables on the one influencing variable is analyzed that is called as multiple regressions. Multiple regression analysis is a technique that allows additional factor to enter the analysis separately so that the effect of each variable can be estimated. It is valuable for quantifying the impact of various simultaneous influences upon a single dependent variable. Because of omitted variables with simple regression, multiple regression analysis is often essential even when the effect of one variable on the influencing variable is to be studied. Multiple regression analysis is capable of dealing with an arbitrarily large number of explanatory variables. Multiple regression analysis is capable of dealing with a randomly large number of explanatory variables. In the present study displacement amplitude of the flexible tube in an array of tube is dependent on the velocity of the fluid (air), type of arrangement of the tube array and pitch over diameter ratio of the array. The displacement amplitude of the tube is dependent variable and velocity of the fluid (air), type of arrangement of the tube array and pitch over diameter ratio are influencing variables. The effect of influencing variable on the dependent variable can be estimated by multiple regression analysis. The effect of influencing variable on the dependent variable can be estimated using experiments also. The experimental results can be compared with the result obtained by the regression analysis.

The data generated from experiments is collected and tabulated using Microsoft Excel software. The graphs are plotted to estimate the effect of influencing variable on dependent variable. The effect of influencing variable is estimated experimentally. Multiple regression analysis is applied on the data with the help of Microsoft Excel worksheet. The relationship between influencing variable and dependent variable is estimated. Relation between displacement amplitude and influencing variables, velocity of the fluid (air), type of arrangement of the tube array and pitch over diameter ratio is estimated. From the experimental data obtained, it is observed that displacement amplitude of the tube is affected by all the three parameters velocity of the fluid (air), type of arrangement of the tube array and pitch over diameter ratio. An empirical relation between displacement amplitude and the influencing variables is obtained.

The values of the influencing variables, velocity of the fluid (air), type of arrangement of the tube array and pitch over diameter ratio can be selected from obtained working range for these parameters. The value for displacement amplitude can be obtained from empirical relation from values of influencing variables, which are selected from the working range. Thus, the predicted value of displacement amplitude is helpful in selecting suitable parameters of the tube array related to vortex induced vibration. The values for displacement amplitude of the tube generated by regression analysis are compared by the values obtained from the experiments conducted. For carrying out the multiple regression analysis, software named DATAFIT is used. Using DATAFIT software, multiple regression analysis is carried out for two different types of arrangement namely inline arrangement and staggered arrangement. Since the type of arrangements does not change with time, this parameter is not considered in the empirical equation developed. The value of displacement amplitude obtained from the empirical relation is shown in the Table 1 for inline arrangement and in the Table 2 for staggered arrangement.

Table -3: Amplitude Displacement of the tube obtained from the empirical relation for inline arrangement

\begin{tabular}{|l|l|l|l|}
\hline Sr. No. & $\begin{array}{l}\text { Velocity } \\
(\mathrm{m} / \mathrm{s})\end{array}$ & $\begin{array}{l}\text { P/d ratio } \\
(\mathrm{mm})\end{array}$ & $\begin{array}{l}\text { Amplitude } \\
\text { Displacement }\end{array}$ \\
\hline 1 & 5 & 2 & 3.24 \\
\hline 2 & 10 & 2 & 6.52 \\
\hline 3 & 15 & 2 & 9.82 \\
\hline 4 & 20 & 2 & 13.13 \\
\hline 5 & 5 & 3 & 2.54 \\
\hline 6 & 10 & 3 & 5.11 \\
\hline 7 & 15 & 3 & 7.7 \\
\hline 8 & 20 & 3 & 10.29 \\
\hline 9 & 5 & 4 & 2.14 \\
\hline 10 & 10 & 4 & 4.3 \\
\hline 11 & 15 & 4 & 6.47 \\
\hline 12 & 20 & 4 & 8.66 \\
\hline
\end{tabular}

Table -4: Amplitude Displacement of the tube obtained from the empirical relation for staggered arrangement

\begin{tabular}{|l|l|l|l|}
\hline $\begin{array}{l}\text { Sr. } \\
\text { No. }\end{array}$ & $\begin{array}{l}\text { Velocity } \\
(\mathrm{m} / \mathrm{s})\end{array}$ & $\begin{array}{l}\text { P/d } \\
\text { ratio } \\
(\mathrm{mm})\end{array}$ & $\begin{array}{l}\text { Amplitude } \\
\text { Displacement }\end{array}$ \\
\hline 1 & 5 & 2 & 4.05 \\
\hline 2 & 10 & 2 & 7.68 \\
\hline 3 & 15 & 2 & 11.17 \\
\hline 4 & 20 & 2 & 14.57 \\
\hline 5 & 5 & 3 & 3.40 \\
\hline 6 & 10 & 3 & 6.45 \\
\hline 7 & 15 & 3 & 9.38 \\
\hline 8 & 20 & 3 & 12.23 \\
\hline 9 & 5 & 4 & 3.00 \\
\hline 10 & 10 & 4 & 5.69 \\
\hline 11 & 15 & 4 & 8.28 \\
\hline 12 & 20 & 4 & 10.80 \\
\hline
\end{tabular}


The results obtained from the empirical relation are represented in the form of graphs to visualize the results and conclude the effect of the influential variables on the dependent variables. The results are plotted for velocity versus displacement amplitude while keeping the pitch over diameter ratio constant and pitch over diameter ratio versus amplitude displacement while keeping the velocity constant.
Following tables represent the amplitude displacement obtained from the multiple regression analysis. In Table 3, the pitch over diameter ratio is kept constant and the amplitude displacement is calculated with respect to change in velocity.

Table -5: Amplitude Displacement for different pitch over diameter ratio obtained from the regression analysis for inline arrangement

\begin{tabular}{|l|l|l|l|l|}
\hline \multirow{3}{*}{ Sr. No. } & $\begin{array}{l}\text { P/d ratio } \\
(\mathrm{mm})\end{array}$ & 2 & 3 & 4 \\
\cline { 2 - 5 } & Velocity(m/s) & Amplitude Displacement & Amplitude Displacement & Amplitude Displacement \\
\hline 1 & 5 & 3.24 & 2.54 & 2.14 \\
\hline 2 & 7 & 4.55 & 3.57 & 3.00 \\
\hline 3 & 9 & 5.86 & 4.60 & 3.87 \\
\hline 4 & 11 & 7.18 & 5.63 & 4.73 \\
\hline 5 & 13 & 8.5 & 6.66 & 5.60 \\
\hline 6 & 15 & 9.82 & 7.70 & 6.47 \\
\hline 7 & 17 & 11.15 & 8.73 & 7.35 \\
\hline 8 & 19 & 12.47 & 9.77 & 8.22 \\
\hline
\end{tabular}

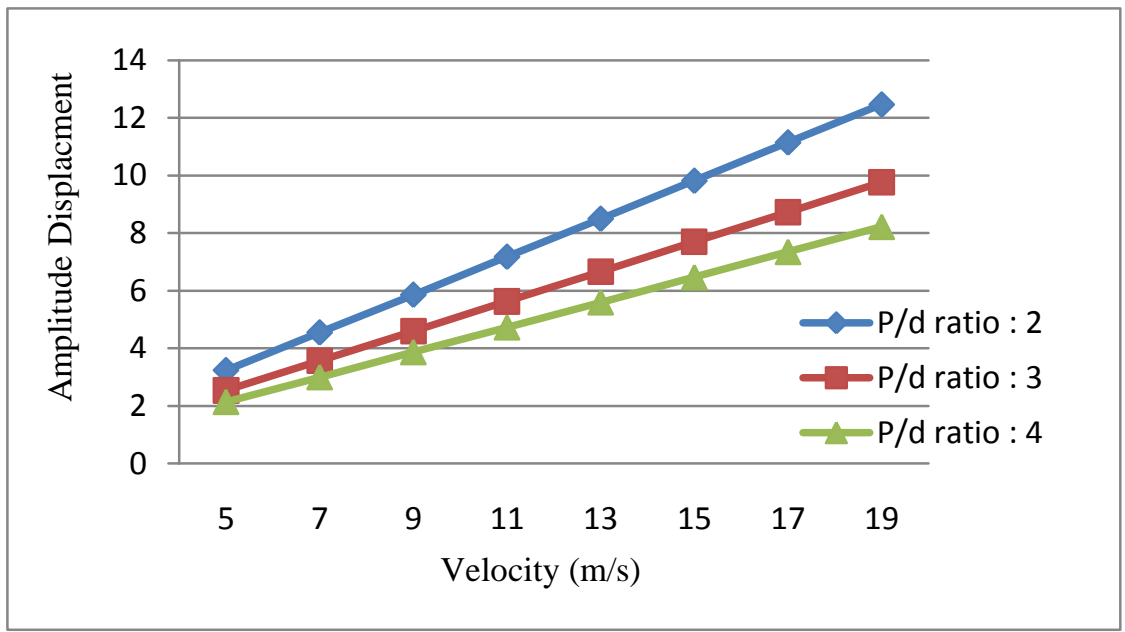

Chart -4: Displacement Amplitude for different pitch over diameter ratio obtained from regression analysis

From the graph, it can be observed that the displacement amplitude of the tube increases with the increase velocity. It can also be seen that maximum amplitude displacement is obtained for pitch over diameter ratio equal to 2. Also the value of amplitude displacement decreases with the increase in the pitch over diameter ratio.

Table -6: Amplitude Displacement for different pitch over diameter ratio obtained from the regression analysis for staggered

\begin{tabular}{|l|l|l|l|l|}
\hline \multirow{2}{*}{$\begin{array}{l}\text { Sr. } \\
\text { No. }\end{array}$} & P/d ratio(mm) & 2 & 3 & 4 \\
\cline { 2 - 5 } & Velocity(m/s) & $\begin{array}{l}\text { Amplitude } \\
\text { Displacement }\end{array}$ & $\begin{array}{l}\text { Amplitude } \\
\text { Displacement }\end{array}$ & $\begin{array}{l}\text { Amplitude } \\
\text { Displacement }\end{array}$ \\
\hline 1 & 5 & 4.05 & 3.4 & 3 \\
\hline 2 & 7 & 5.52 & 4.64 & 4.09 \\
\hline 3 & 9 & 6.97 & 5.85 & 5.16 \\
\hline 4 & 11 & 8.39 & 7.04 & 6.22 \\
\hline 5 & 13 & 9.79 & 8.21 & 7.25 \\
\hline 6 & 15 & 11.17 & 9.38 & 8.28 \\
\hline 7 & 17 & 12.54 & 10.52 & 9.29 \\
\hline 8 & 19 & 13.90 & 11.66 & 10.3 \\
\hline
\end{tabular}




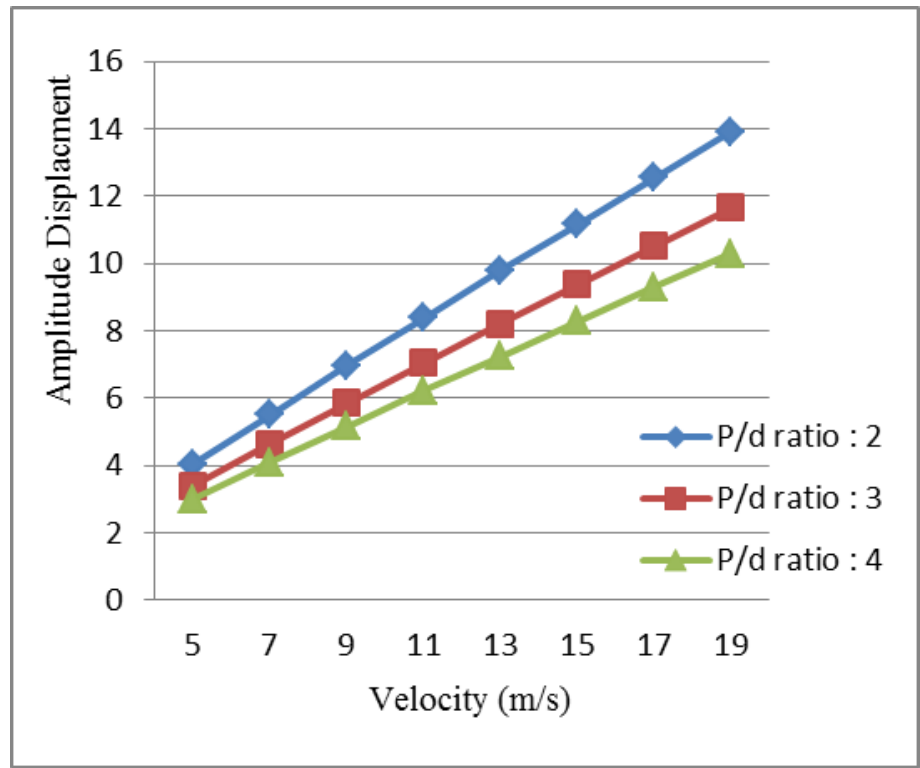

Chart -5: Displacement Amplitude for different pitch over diameter ratio obtained from regression analysis

From the graph, it can be observed that the displacement amplitude of the tube increases with the increase velocity. It can also be seen that maximum amplitude displacement is obtained for pitch over diameter ratio equal to 2 . Also the value of amplitude displacement decreases with the increase in the pitch over diameter ratio.

\section{CONCLUSION}

Experiments are carried out on inline and staggered arrangement of tube arrays. For inline arrangement, nine tubes are used and the amplitude displacement of the center tube is found out with the help of the load cell arrangement. For staggered arrangement, eight tubes are used and the amplitude displacement of the tube on the right side from the direction of the flow is considered. For both, inline and staggered arrangement, amplitude displacement is measured for three different pitches over diameter ratios $(\mathrm{p} / \mathrm{d})$, which are 2, 3 and 4 .

Following results are concluded based on the results of the experiments conduced

1. It is observed from the graphs that the results obtained from the experiments conducted and the results obtained from the regression analysis are in close agreement with one another

2. For inline arrangement, maximum amplitude displacement is measured for the $\mathrm{p} / \mathrm{d}$ ratio equal to 2. The value of amplitude displacement goes on decreasing as the value of $\mathrm{p} / \mathrm{d}$ ratio increases from 2 to 4. Similar results are observed in case of regression analysis.

3. For staggered arrangement, results similar to inline arrangement are observed i.e. the maximum value is measured for $\mathrm{p} / \mathrm{d}$ ratio equal to 2 and the value of displacement goes on decreasing as the p/d ratio increases from 2 to 4 . As we can observe from chart 5 , it can be observed that similar trend is observed. Also maximum displacement amplitude is obtained for $\mathrm{p} / \mathrm{d}$ ratio equal to 2 .
4. When the results are compared for staggered and inline arrangement, the value of displacement amplitude for staggered arrangement is more compared to that of inline arrangement. As seen in the table, for $\mathrm{P} / \mathrm{D}$ ratio $=2$, the maximum value of displacement amplitude is $15 \mu \varepsilon$ for staggered arrangement while for inline arrangement the maximum value is $13 \mu \varepsilon$. Also in regression analysis the results are in close agreement.

5. It can be concluded that maximum displacement is obtained in staggered arrangement and it is obtained in the tube array with least pitch over diameter ratio.

\section{REFERENCES}

[1] H. G. Goyder, "Flow Induced Vibration in Heat xchangers", Trans IChemE, Volume 80, Part A, April 2002.

[2] S. Pasto. "Vortex-induced vibration of a circular cylinder in laminar and turbulent flow", Journal of Fluids and structures, 24, 977-993, 2008.

[3] Hardik R Gohel, Balkrushna A Shah, Absar M Lakdawala - Numerical Investigation of Flow Induced Vibration for the Triangular Array of Circular Cylinder'.

[4] F. J. Huerta-Haurte, M. Gharib, "Flow Induced Vibration of a side-by-side arrangement of two flexible circular cylinders", Journal of Fluids and Sturctures 27, 354-366.

[5] T. K. Prasanth, Sanjay Mittal, "Vortex-induced vibration of two circular cylinders at low Reynolds number", Journal of Fluids and Structures, 25, 731741, 2009.

[6] Chunlei Liang, George Papadakis, Xiaoyu Luo, "Effect of tube spacing on the vortex shedding characteristics of laminar flow past an inline tube array: A numerical study",computers and fluids, 38,950-964,2009. 
[7] M. P. Paidoussis, "Real-Life experiences with flow induced vibration", Journal of Fluids and Structures, Volume 22, April 2006.

[8] E. Longette, Z. Bendjeddou, M. Souli, "Methods for numerical study of tube bundle vibrations in crossflows", Journal of Fluids and Structures, 18, 513528, 2003.

[9] P. A. Feenstra, D. S. Weaver, T. Nakamura, "Vortex shedding and Fluidelastic instability in a normal square tube array excited by two phase cross flow", Journals of Fluids and Structures, 17, 793-811, 2003.

[10] Grover L. K., D. S. Weaver, "Cross-Flow Induced Vibration in a Tube Bank-Vortex shedding", Journal of Sound vibration, 59, 263-276.

[11] T.L. Morse, R.N.Govardhan, C.H.K.Williamson 'The effect of end conditions on the vortex-induced vibration of cylinders'.

[12] Shahab Khushnood, Zaffar M. Khan, M. Afzaal Malik, Zafar Ullah Koreshi, Mahmood Anwar Khan, "A review of heat exchanger tube bundle vibrations in two-phase cross-flow", Nuclear Engineering and Design, 230, 233-251,2004.

[13] S. Pasto. "Vortex-induced vibration of a circular cylinder in laminar and turbulent flow", Journal of Fluids and structures, 24, 977-993, 2008.

[14] K.Lam, G. D. Jiang, Y. Liu, R. M.C. So, "Simulation of cross flow induced vibration of cylinder arrays by surface vorticity method", Journal of Fluid and Structures, 22(2006), 1113-1131.

[15] Granger, S., Campistron, R., and Lebret, J., 1993, "Motion-Dependent Excitation Mechanisms in a Square In-Line Tube Bundle Subject to Wear CrossFlow: An Experimental Modal Analysis," Journal of Fluids and Structures, 7, 521-550.

[16] Robert D. Blevins, "Flow Induced Vibration",Second Edition.

\section{BIOGRAPHIES}

Vimal D Tandel is currently pursuing M.E in design engineering at Sinhgad Institute of Technology and Science, Narhe, Pune affiliated to Pune University. He has completed B.E in Mechanical engineering from Gujarat Technological University in 2012.

Rajesh V Patil is currently working as Assistant Professor in the department of Mechanical engineering in Sinhgad Institute of Technology and Science, Narhe, Pune. 